\title{
Scrivimi ancora, arrivederci. Roma Correu
}

Ludovica Marinaro

Università degli studi di Firenze, DIDA, Italia

Ludovica.marinaro@unifi.it 
Ho potuto vedere così, come se non l'avessi mai visto prima, un lembo di città senza il movimento perpetuo quotidiano, senza le auto in sosta, senza persone, senza suoni e rumori. Ho visto l'architettura riproporsi nella sua essenza, filtrata dalla luce, in modo sorprendentemente scenografico e monumentale. (Gabriele Basilico, 2007)

Le parole di Gabriele Basilico descrivono alla perfezione quanto a circa quarant'anni di distanza è accaduto ad Arsenio Pistone, autore di questo piccolo prezioso volume, Roma Correu, viatico per immagini che ritrae la città di Roma durante il primo lockdown nella primavera 2020. Un parallelo calzante con una sostanziale differenza. Quella che per il fotografo milanese era una conquista, condizione straordinaria ricercata accuratamente nelle ore assolate della domenica di Pasqua del 1978, si è data invece come un'imposizione, nuova condizione globale della città durante la pandemia di Covid-19. La città vuota. Improvvisamente inaccessibile, lo spazio urbano si è trasformato privandoci del nostro consueto ruolo di attori e relegando la maggior parte di noi nella sola dimensione di spettatori attoniti. Tra i pochi eletti a poter (dover) uscire, Arsenio, può ancor meglio sfoggiare le note cangianti della sua veste double-face di postino e al contempo scenografo, come Fabio Manfredi e Antonio Dattilo elegantemente sottolineano nel loro accompagnamento al periplo quotidiano dell'amico.
Quella che per il postino era la quotidianità del lavoro, routine abitudinaria di slalom speciale sulle dolci pendenze del centro storico tra moltissime persone, si è trasformata nel viaggio inatteso dello scenografo su un palco vasto e deserto che lo ha visto protagonista. Un cambio di focale netto, che ha sovvertito la percezione dello spazio e del senso dei luoghi, consentendo ad Arsenio, e a tutti attraverso le sue cartoline dal confine, di sviluppare una ri-cognizione della città, nell'interezza delle sue sfumature semantiche. In tal senso Roma Correu è il diario essenziale di un vóotos contemporaneo, perché compiuto in momento in cui per ognuno di noi la tensione tra 'dentro' e 'fuori' si era fatta palpabile, ed il 'fuori', lo spazio pubblico era visto e attraversato non senza un inedito timore, così ferocemente contrapposto alla sicurezza ed intimità del "dentro" delle nostre case. Di qui emerge il potere insolito di questo piccolo libro pubblicato dalla casa editrice catalana Úrsula llibres, grazie al coraggio di Robert Fabregas, direttore della Casa de la Cultura di Salt, Les Bernardes, che ha giustamente intuito che parlare di Roma non è che un pretesto, peraltro molto pertinente, per parlare della Città: L'Eterna Urbe ancora una volta come paradigma di un discorso molto più ampio, globale, su cosa sia accaduto alla Città durante la prima reclusione e di come ne sia uscita trasformata. 
Parlo di un potere insolito perché il libro riesce a dare coerente e simultanea rappresentazione a coppie di concetti antitetici: locale e globale, come già accennato, ma anche interno ed esterno, pieno e vuoto, prendendo le sembianze di un racconto al contempo intimo e universale, finito e aperto. È esso stesso elastico. Così come il tempo e lo spazio che descrive, proprietà che emerge chiaramente ora attraverso la penna agile di Daniela Colafranceschi nella sua introduzione, ora senza mediazione dagli scatti e dal pentagramma di riflessioni che li accompagnano. Girato, per così dire, in presa diretta, Roma Correu fonde due strumenti e due prospettive, quella dei narratori che offrono visioni protese dalla dimensione domestica materializzate negli scritti che scandiscono la storia, e quella en plein air dalla vespa di Arsenio che prende forma attraverso le immagini scattate durante gli itinerari di consegna ai codici postali 00186 e 00187. Ciò che ne risulta è maggiore della semplice sommatoria delle parti e si compone in maniera essenziale dello stesso sguardo e della memoria di chi legge, chi insieme all'autore viaggia, vedendo finalmente ciò che per quei mesi era negato e mancava, o con gli spettatori attende, ha atteso e lascia che le riflessioni maturate in quei momenti riaffiorino e si imprimano sul pentagramma. Questo piccolo libro ha il duplice valore di testimonianza di un'esperienza "finita", quella di un gruppo ristretto di persone e di peripli ben circoscritti, e quello di scrigno gentilmente offerto ai ricordi e alle sensazioni del lettore, lasciato libero di riempire il vuoto percepito di questi spazi urbani, di compiere voli pindarici verso tutte le altre città, proprietà che ne fa dunque un progetto aperto e al contempo un luogo di incontro.

Vi è poi un aneddoto che rende a mio avviso ancora più interessante leggere ed esplorare i molteplici significati di questo progetto.

Gli autori, attore-scenografo e spettatori-registi, sono amici e tre di loro sono vicini di casa a Roma. Questo, che potrebbe sembrare un dettaglio futi-

le, concorre a nutrire un'essenziale chiave di lettu- ra di un periodo così unico e controverso, come è stato il primo lockdown, oggi che viviamo l'imperativo necessario della transizione ecologica. Testimonia l'importanza e l'urgenza di intraprendere questa sfida abbracciando un approccio paesaggistico all'interpretazione e alla trasformazione della realtà, ovvero dando corpo ai principi espressi dalla Convenzione Europea del Paesaggio, che proprio I'anno scorso ha compiuto vent'anni senza esser ancora pienamente attuata. In un momento in cui così forte e sofferta era la sensazione di privazione di una dimensione essenziale a definire la nostra stessa identità, sociale e culturale, in cui lo spazio pubblico e la città mai ci sono sembrati così distanti, è stata la ricerca appassionata di una visione condivisa, inclusiva, sinestetica e sintetica a rinsaldare quel legame reciso violentemente il 9 marzo 2020, consentendo di continuare a produrre paesaggio. La coesione e la progettualità di una piccolissima comunità, che ha redistribuito e condiviso tra i suoi componenti 'i sensi' per percepire e descrivere la realtà, ha fatto in modo che non vi fosse interruzione nella scrittura-lettura continua del paesaggio (come opera collettiva) e che da questa si potesse, oggi più che mai, trarre insegnamento. Se possibile questo libro riesce così in un'ulteriore proficua fusione, quella di due generi fotografici, quello di reportage, o documentaristico, e quello di paesaggio, urbano, e quindi in questo caso quella che proprio Basilico ha definito "nuova fotografia di paesaggio". Dati il pathos, che indiscutibilmente associamo a quel momento dell'emergenza, e il 'vuoto' con cui si è manifestato, gli scatti rappresentano entrambe le tradizioni ed i generi. Ritraggono una condizione di contingenza estrema che assume però una posa estatica e perenne, una realtà apparentemente immobile eppure in tensione. La città vuota lo è stata poi veramente?

Complice una prassi consolidata in cui il vuoto si dà come semplice residuo di una certa tendenza pianificatoria impegnata a definire esclusivamente il pie- 


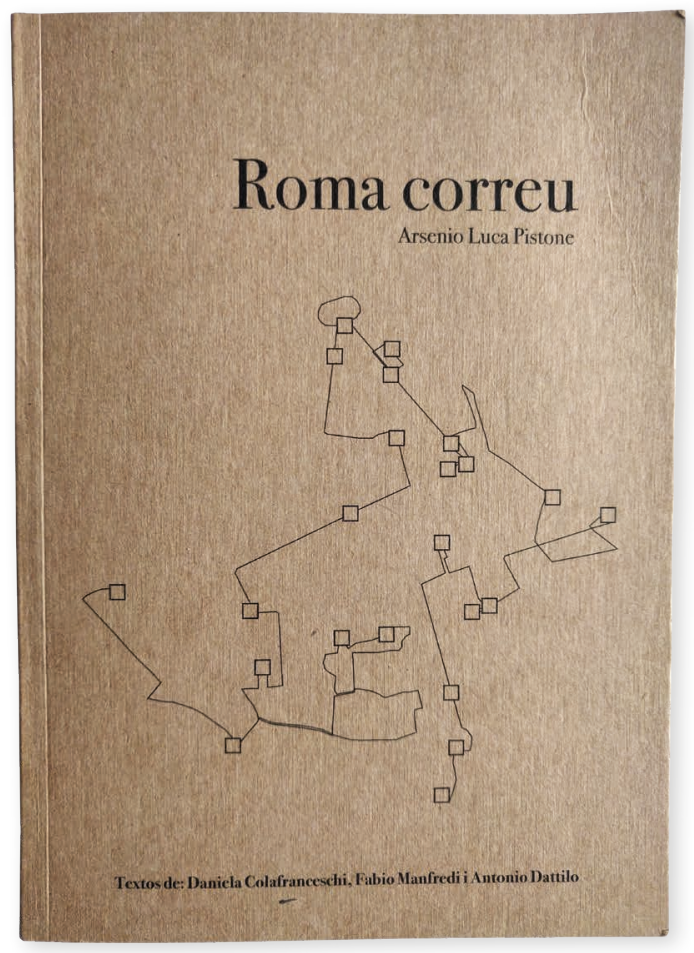

no, la città ha sottovalutato il peso e il ruolo del vuoto nella costruzione della sua immagine, riducendone anche il significato: la città è vuota quando ne è assente l'uomo. Ed osservandola per la prima volta, effettivamente spoglia del nostro turbinio, ci siamo illusi inizialmente potesse essere davvero così. Ma "Il vuoto per me non significa mai vera assenza: si tratta piuttosto di una fase di silenzio che mi permette di instaurare un dialogo spero autentico con la realtà. (...) l'assenza dell'uomo toglie al vuoto la dimensione d'angoscia e fa del vuoto ciò che veramente è". Prendo ancora in prestito le parole di Gabriele Basilico dall'opera Dentro la città, affinché possano guidarci nel rovesciamento di questa concezione ostinatamente antropocentrica fino alla conquista di una prospettiva più inclusiva e attenta alla complessità del mondo vivente, fatto anche di fauna e soprattutto di piante. È ciò che ci è accaduto durante la reclusione, quando abbiamo visto tassi, cinghiali e caprioli avventurarsi tra le vie libere delle nostre città, e che Arsenio Pistone ha documentato nei suoi scatti, quando osservava un prato pioniero riempiere l'ordito e trama continui dei sanpietrini di Piazza del Popolo. La città senza di noi si è mostrata pronta e disponibile ad accogliere presenze plurali, con ritmi e passi differenti, pronta alla contaminazione e ad essere reinventata, decisa a rigenerarsi e non più ad espandersi. Dobbiamo cogliere questo invito, ricordarne la pacata eleganza. Queste ed altre riflessioni è capace di creare questo prezioso libro. Cercando di evitare il paradosso in cui una recensione possa superare in parole spese ciò che il libro stesso non ha volutamente scritto ma ha evocato, lascio ai futuri lettori il compito di contribuire a questa scrittura corale aperta, ricordando tutte le volte che in quella primavera, guardando la nostra città dalla finestra le abbiamo rivolto un affettuoso: arrivederci. 